\section{A COMBINATION OF FUNCTIONAL BIOMARKERS IMPROVES IDENTIFICATION OF THE TUMOR-SPECIFIC REACTIVE T CELL REPERTOIRE}

${ }^{1}$ Arianna Draghi*, ${ }^{2}$ Katja Harbst, ${ }^{1}$ Inge Svane, ${ }^{1}$ Marco Donia. ${ }^{1}$ Copenhagen University Hospital, HERLEV, Denmark; ${ }^{2}$ Lund University, Lund, Sweden

Background Detecting the entire repertoire of tumor-specific reactive $\mathrm{T}$ cells is essential for investigating the broad range of $\mathrm{T}$ cell functions in the tumor-microenvironment. At present, assays identifying tumor-specific functional activation measure either upregulation of specific surface molecules, de novo production of the most common antitumor cytokines or mobilization of cytotoxic granules.

Methods In this study, we combined transcriptomic analyses of tumor-specific reactive tumorinfiltrating lymphocytes (TILs), TIL-autologous tumor cell co-cultures and commonly used established detection protocols to develop an intracellular flow cytometry staining method encompassing simultaneous detection of intracellular CD137, de novo production of TNF and IFNy and extracellular mobilization of CD107a.

Results This approach enabled the identification of a larger fraction of tumor-specific reactive $\mathrm{T}$ cells in vitro compared to standard methods, revealing the existence of multiple distinct functional clusters of tumor-specific reactive TILs. Publicly available datasets of fresh tumor single-cell RNA-sequencing from four cancer types were investigated to confirm that these functional biomarkers identified distinct functional clusters forming the entire repertoire of tumor-specific reactive $\mathrm{T}$ cells in situ.

Conclusions In conclusion, we describe a simple method using a combination of functional biomarkers that improves identification of the tumor-specific reactive $\mathrm{T}$ cell repertoire in vitro and in situ.

http://dx.doi.org/10.1136/jitc-2020-SITC2020.0762

\section{EXPANDED AND ACTIVATED TILS KILL TUMOR CELLS ENABLING IO COMPOUND ASSAYS}

${ }^{1}$ Paula Comune Pennacchi, 2Paloma Navarro, 'Verónica García Navas, ${ }^{2}$ Arántzazu Barquín García, ${ }^{2} E l e n a$ Sevillano Fernández, ${ }^{2}$ Sergio Ruiz Llorente, ${ }^{2} J u a n$ Francisco Rodriguez, ${ }^{2}$ Monica Yagüe, ${ }^{1}$ Joan Ballesteros*, ${ }^{1}$ Daniel Primo, ${ }^{2}$ Jesus Garcia Donas Jimenez. ${ }^{\prime}$ Vivia Biotech, Tres Cantos, Madrid, Spain; ${ }^{2}$ HM Hospitales - Centro Integral Oncológico HM Clara Campal, MADRID, Spain

Background The Tumor Microenvironment (TME) has a key role in solid tumor therapy screening. We have developed a $3 \mathrm{D}$ ex vivo immunosuppressive assay mimicking the TME. It enables both allogenic \& autologous tumor lysis by expanded Tumor Infiltrate Lymphocytes (TILs). It is a valuable 3D assay to study the activity of immune therapy drugs in patient sample.

Methods TME-aligned immunosuppressor media was produced by conditioned media from activated human Mesenchymal Stem Cells (hMSC). The TILs were expanded from patientderived tumor samples and used for tumor killing potential evaluation. Target tumor cells were obtained from different sources: a) Isolated from patient-derived material and frozen until use in experiments with autologous or allogenic TILs or b) Tumor cell lines purchased from ATCC. The cells were mixed according to desired Effector:Target (E:T) ratios and embedded in 3D matrix in presence of TME-aligned media and immune therapy compounds, as Immune Checkpoint inhibitors (ImmChPi). The cell retrieval was performed at the end of desired timepoints and tumor cell killing and TILs activation profile were analyzed by flow cytometry.

Results The in vitro expanded TILs were able to kill autologous and allogenic tumor cells in several different $\mathrm{E}: \mathrm{T}$ ratios within 24 hours. The $\%$ tumor cell killing for allogenic samples of the same cancer type showed a similar range as autologous killing. In a representative autologous $\mathrm{E}: \mathrm{T}$ experiment we observed $40 \%$ of killing at E:T ratio 10:1 (figure 1A). These same TILs showed even higher\% tumor killing against allogenic tumor samples (up to 90\%, data not shown). The Immune Check Point (ImmChP) expression during expansion may change and was followed to select proper expansion timelines. For example, in a particular ovarian cancer sample TIM3 was expressed in 75\% of the expanded TILs (figure $1 \mathrm{~B})$ and the treatment with TIM3 blocking antibody increased nearly 2-fold tumor cell killing in a dose dependent manner (figure 1C).

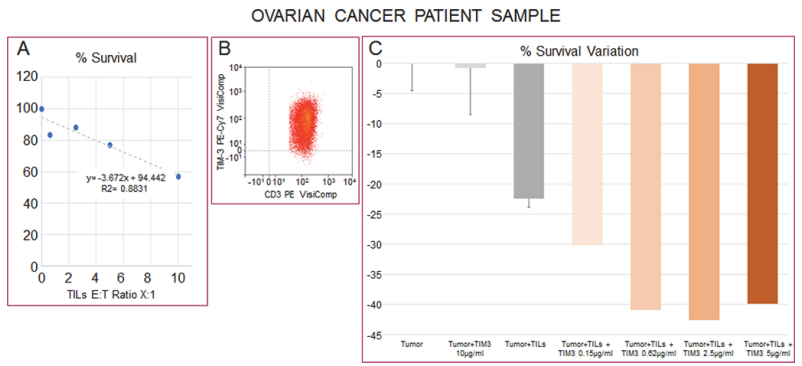

Abstract 763 Figure 1

Autologous killing: novel ex vivo solid tumor 3D assay using autologous TILs from an ovarian cancer patient sample for Immune Check Points or other IO drugs. A) Autologous TILs kill 40\% ovarian cancer cells in $24 \mathrm{~h}$ incubation. B) $75 \%$ TILs express TIM3. C) Adding TIM3 antibody enhances killing of tumor cells in a dose dependent manner.

Conclusions The Novel TME-Aligned 3D IO Assay is a reliable, and powerful tool to study the mode of action of tumor cells lysis by expanded TILs. Immune Therapy Drugs Screening can be performed in autologous or allogenic E:T conditions, allowing full mode of action description of $\mathrm{Bi}$ or Multispecific antibodies, ImmChPI and others, and opens a new door for therapy prediction studies in patient's material.

Ethics Approval The study was approved by Hospital 12 de Octubre Ethics Board, with approval number 14/199.

Consent Written informed consent was obtained from the patient for publication of this abstract and any accompanying images. A copy of the written consent is available for review by the Editor of this journal.

http://dx.doi.org/10.1136/jitc-2020-SITC2020.0763 